\title{
Los componentes de la imagen de la nación como base para formulación de la identidad competitiva: el caso del Perú
}

\author{
Gina María Pipoli de Azambuja \\ Candidata a Doctora en Economía y Dirección de Empresas por la Universidad de Deusto, \\ San Sebastián, España. \\ Magíster en Administración por la Universidad del Pacífico, Lima, Perú. \\ Profesora e Investigadora del Departamento Académico de Administración de la \\ Universidad del Pacífico, Lima, Perú. \\ Juan Alejandro Flores Castro \\ Doctor en Economía y Dirección de Empresas por la Universidad de Deusto, Bilbao, España. \\ Master en Administración de Empresas por la Universidad de Deusto, Bilbao, España \\ Profesor e Investigador del Departamento Académico de Administración de la \\ Universidad del Pacífico, Lima, Perú.
}

\section{Resumen}

En el mundo globalizado en el que vivimos, hoy, los países pugnan por diferenciarse unos a otros como mecanismo para poder atraer más turistas, incrementar el volumen de sus exportaciones y captar más inversiones, de manera que puedan tener mayores fuentes de ingresos. Para ello, buscan lograr una diferenciación en el mercado, a partir de los elementos que constituyen su identidad nacional, la cual influirá en la percepción de la imagen de la nación del consumidor y será la base para generar su identidad competitiva, que constituirá la piedra angular sobre la que se diseñará la estrategia de marketing internacional de los países para salir a competir en el mercado internacional.

Es así que la identidad competitiva se ha convertido en una pieza clave para promover la competitividad nacional, al sintetizar la imagen de la nación mostrada al mercado para cumplir sus objetivos. Por tal motivo, el objetivo de este trabajo es identificar los elementos de la imagen nación Perú, a partir de la identidad nacional percibida por los consumidores, tanto nacionales como extranjeros, para que sobre esa base se diseñe una estrategia de identidad competitiva para el Perú.

\section{Palabras clave}

Estrategia de posicionamiento, Identidad nacional, Identidad competitiva.

\section{Introducción}

A lo largo de las últimas décadas, se han producido ciertos cambios en las tendencias de internacionalización de las empresas que buscan poder satisfacer las diversas necesidades de los consumidores en un mercado cada vez más globalizado. En este contexto, el comercio internacional se constituye en una importante herramienta, en la que convergen la oferta y demanda 
global (Mohamad et al. 2000). De esta manera, frente a un mercado cada vez más amplio, con una oferta igualmente extensa y gran variedad de productos y servicios, las empresas con presencia en diversos países se han visto obligadas a modificar sus estrategias de posicionamiento, con miras a captar la atención, el interés y la preferencia de sus consumidores provenientes de mercados extranjeros (Porter 1998). No obstante, además de las organizaciones, es importante reconocer que, en un nivel macro y en un contexto global, los países compiten día a día por incrementar su capacidad de influir en el comportamiento de los consumidores extranjeros, ya sea mediante un incremento de sus exportaciones, la atracción de nuevos turistas o la captación de mayores inversionistas para su desarrollo y crecimiento (Vanossi et al. 2006). Sin embargo, para poder lograr el alineamiento y un efecto de sinergia positiva de estas estrategias, se requiere que tanto el sector público como el privado integren sus esfuerzos y trabajen coordinadamente para alcanzar el posicionamiento buscado en el mercado externo (Madhavaram et al. 2005). Así, la estrategia de internacionalización se convierte en una herramienta fundamental para poder construir la imagen de un país.

Desde el punto de vista de un país, este proceso de construcción de la identidad competitiva puede resultar aun más complicado. Por tanto, se requiere de una visión de largo plazo para cosechar los frutos de un trabajo adecuado en el desarrollo de una estrategia de internacionalización.

En un mundo global y competitivo, las naciones desarrollan una ventaja competitiva con la finalidad de presentarse como la mejor opción ante las decisiones del consumidor internacional, por lo que su espectro de actuación para definir tal estrategia se enmarca, entre otros aspectos, en la generación de un ambiente propicio para la inversión extranjera, directa o indirecta; en las políticas públicas de fomento al comercio exterior -pasando por los tratados bilaterales de libre comercio-; y, como una derivación de la primera opción, en el desarrollo de un sector servicios, que genera una inversión directa a baja escala -si se le analiza de manera desagregada-, pero de grandes dimensiones si se procede a evaluar su impacto en la economía en el nivel de grandes agregados macroeconómicos. Nos estamos refiriendo a la promoción del sector turismo (Dinnie 2008). No obstante, algunos investigadores añaden objetivos adicionales a los señalados, como la estabilidad política, la recuperación de la credibilidad internacional, el estímulo de acuerdos comerciales, la atracción de emprendedores, entre otros (Temporal 2002, Vanossi 2006). Enmarcan el tema más hacia la «identidad competitiva» (Anholt 2007) y, por lo tanto, a destacar la ventaja competitiva que la marca puede proporcionar a una determinada nación, en función del posicionamiento que se quiera lograr en el mercado global.

Existe abundante literatura sobre el concepto de imagen país e identidad nacional. A lo largo de las últimas décadas, se ha investigado mucho sobre estos temas, tratando de encontrar la vinculación de estos dos conceptos y su relación de causalidad-efecto. Sin embargo, no existe mayor información sobre el diseño de las estrategias de marketing internacional de los países a partir de los componentes de su imagen y su vinculación con su identidad nacional.

Por tanto, este trabajo de investigación pretende identificar los elementos de la imagen nación Perú, a partir de la identidad nacional percibida por los consumidores, tanto nacionales como extranjeros, para que sobre esa base se diseñe una estrategia de identidad competitiva para el Perú.

Es así que en la primera parte del trabajo se presentan los resultados de la revisión de la literatura que sustentan el marco conceptual propuesto, analizando para ello los componentes de la imagen de la nación y la influencia de esta en la percepción y evaluación del público extranjero. En la segunda parte del documento, se presenta el trabajo empírico para determinar los componentes de la imagen del Perú, realizado sobre la base de 356 encuestas aplicadas al público de tres países: Perú, Estados Unidos y Francia. 
Los resultados del trabajo empírico nos muestran los componentes de la imagen nación Perú, a partir de los cuales se deberá construir la estrategia de identidad competitiva.

\section{Objetivo de la investigación}

El objetivo general de la presente investigación es identificar los elementos de la imagen nación Perú, a partir de la identidad nacional percibida por los consumidores, tanto nacionales como extranjeros, para que sobre esa base se diseñe una estrategia de identidad competitiva para el Perú.

\section{Marco conceptual}

Durante los últimos años, las instituciones y organizaciones se han abocado al desarrollo de estrategias de posicionamiento, dado que los países hoy en día son conscientes de que están compitiendo con otras naciones por captar mayores recursos con el fin de mejorar su desarrollo económico.

En este sentido, los países desarrollan, intencionalmente o no, estrategias de marketing que superen las de su competencia y les permitan tener la preferencia del consumidor internacional (Kotler et al. 1993).

Según Dinnie (2008), en la construcción de la imagen de la nación, los países se focalizan en diversos componentes y elementos de comunicación de su identidad, en aquellos que sean los mas apropiados para alcanzar los objetivos específicos de identidad nacional. Es así que los países se pueden beneficiar de estrategias de exportaciones y, por tanto, incorporar el elemento comercial de su oferta exportable en la estrategia de comunicación de su identidad nacional; mientras que otros países podrán focalizarse más en sus logros deportivos o en su oferta turística. Cualquiera que sea la estrategia elegida, implicará un efecto importante en la imagen de la nación, la cual es hoy de suma importancia para los países que compiten en un mundo globalizado (Leclere et al. 1994).
A partir de estos elementos se presenta un modelo conceptual, desarrollado sobre la base de la propuesta de Dinnie (2008). El modelo que proponemos contempla una relación dinámica entre los constructos de identidad nacional, imagen de la nación e identidad competitiva, y muestra cómo la imagen de la nación se deriva como una consecuencia de la identidad nacional, la cual se da a conocer por diversos medios, como las expresiones culturales, elementos de marca, estrategias de marketing, entre otros. El modelo contempla que a partir de las variables que integran la imagen nación se generará la identidad competitiva.

La identidad competitiva es la resultante de la conjunción de la imagen de la nación que interactúa con variables de competitividad de índole económica y política, además de las tradicionales variables comerciales de marca, que generan un posicionamiento diferenciador y distintivo entre una serie de alternativas para el consumidor.

Por este motivo, Anholt (2007) señala que las naciones buscan desarrollar una determinada identidad competitiva para alcanzar objetivos esenciales que permitan competir en un mundo globalizado, como es el caso del fomento del turismo, dada la importancia que tiene este sector en el desarrollo de la economía de los países.

Adicionalmente, Temporal (2002) sugiere que además de las metas fundamentales vinculadas a la atracción de turistas, entre otras, la estrategia de posicionamiento del país debe ser capaz de favorecer la estabilidad monetaria, ayudar a mejorar la credibilidad internacional, revertir los aspectos negativos que pueden percibirse en el mercado externo, incrementar la influencia política del país en el mundo, promover los acuerdos comerciales con importantes socios estratégicos y, por último, hacer más sólido e interiorizar el concepto de «nación» entre toda la población.

Desde otro punto de vista, Ollins (2002) lleva a cabo un análisis desde una perspectiva de evo- 
lución histórica de las estrategias de posicionamiento, y señala que lo único nuevo en torno a una estrategia de posicionamiento de los países es la terminología utilizada en la actualidad, pues las naciones siempre se han distinguido unas de otras por medio de símbolos, moneda, himnos, nombres, etcétera. Sin embargo, lo que Ollins no contempla en su análisis es la posición estratégica que una nación puede alcanzar más allá de los símbolos que la identifican frente a otras naciones.

\section{La identidad nacional como componente de la estrategia de posicionamiento}

La identidad puede definirse como lo que realmente es; es decir, la esencia de algo o alguien; mientras que la imagen se refiere a cómo ese algo es percibido, lo cual ciertamente refleja un carácter bastante subjetivo. Así, la brecha existente entre ambos conceptos; identidad e imagen, puede representar un factor negativo, pues en muchas ocasiones los países son percibidos por lo que no son en realidad. Esto último demuestra una clara distorsión entre la identidad y la imagen, lo cual permite verificar el carácter subjetivo atribuido a la imagen (O'Shaughnessy y Jackson 2000, Kotler y Gertner 2002).

Para que sea exitosa, el desarrollo de una estrategia de posicionamiento de un país debe comenzar por la construcción de una identidad competitiva. Este es un proceso complejo, pues se deben identificar qué elementos, de los múltiples que pueden incluirse dentro de la identidad de una país, son importantes y favorables para los objetivos planteados en la campaña de desarrollo de la estrategia del país (Dinnie 2008).

Es importante comprender y valorar las distintas características que definen la identidad de un país, en vista de que, finalmente, a partir de esta se elabora la estrategia de posicionamiento de un país (Howard 1989). Entre los varios componentes que definen la identidad de un país, destacan la geografía, así como su historia, sus manifestaciones culturales, su música, sus ciuda- danos ilustres o no ilustres y la iconografía expresada por los símbolos patrios (como la bandera nacional) (Smith 1991, Tuck 2003). Respecto de las manifestaciones culturales, Anderson (1991) incluye dentro de estas a la poesía, literatura, música y demás artes. Desde otro punto de vista, Shulman (2002) define como componentes culturales a la lengua de una nación, así como su religión y tradiciones.

Luego de definir los elementos que conforman la identidad competitiva de un país, se debe analizar cómo proyectar esta información al resto del mundo por medio de la estrategia de posicionamiento de un país.

El primer paso es reconocer lo que representa la identidad de un país, para luego valorar la importancia de dicha identidad dentro de la estrategia de posicionamiento del país (Jaworski y Fosher 2003). La identidad de un país representa un concepto abstracto. Sin embargo, las distintas investigaciones desarrolladas en torno a este tema, generalmente, convergen en una definición bastante similar, aunque en función de distintos puntos de vista pueden establecerse diferencias. Kotler y Gertner (2002) descomponen el concepto y señalan que la identidad del país representa un conjunto de asociaciones e información relacionada con una nación determinada, pero de manera simplificada, como resultado de un proceso mental, al tratar de acopiar la información más relevante sobre este lugar.

Los componentes que construyen la identidad nacional son: geografía política, relaciones internacionales, ciencia política, antropología cultural, psicología social, leyes internacionales, sociología e historia; elementos que permiten a los consumidores diferenciar un país de otro a partir de sus componentes esenciales (Dinnie 2008).

Sin embargo, al ser un concepto abstracto, la identidad nacional puede recibir múltiples y distintas interpretaciones en cada nación alrededor del mundo y aún más, puede variar en la percepción de cada ciudadano. Esto es posible porque cada 
individuo en el mundo tiene la capacidad de concebir la realidad de una forma única y diferente al resto.

Finalmente, es necesario considerar, además de la identidad nacional, el posicionamiento de la imagen del país en la mente del consumidor, sin el cual todos los esfuerzos por desarrollar una estrategia de identidad competitiva no tendría un resultado positivo. De este modo, el posicionamiento de un país depende intrínsicamente de dos procesos previos sumamente importantes y sin los cuales las estrategias derivadas de este objetivo serían inconsistentes; la diferenciación y la segmentación (Keller 2003, Bauer et al. 2006).

En el gráfico 1 se presenta el marco conceptual que se ha elaborado, luego de la revisión de la literatura, para mostrar los constructos identificados, así como sus relaciones. Se observa que los indicadores de la identidad nacional son los factores geográficos, políticos, socioculturales, relaciones internacionales e historia; mientras que los dos indicadores de la imagen país serían los componentes cognitivos y los componentes afectivos.
La identidad nacional influenciaría en la imagen de la nación y esta, a su vez, tendría incidencia en la identidad competitiva del país en el mercado internacional, por lo que las estrategias de marketing internacional tendrían como eje central a la identidad competitiva cuando entren a competir en mercados extranjeros.

\section{Metodología}

Para realizar el análisis empírico, se han evaluado las percepciones de los consumidores de tres países correspondientes a tres regiones geográficas distintas, como los Estados Unidos, Francia y el Perú, de modo que estén representados tres grupos de tres países con distinto nivel de flujo turístico y conocimiento del Perú.

Es así que se eligió como primer mercado a los Estados Unidos, debido a que, según el estudio sobre el Perfil del Turista Extranjero 2007 elaborado por PromPerú ${ }^{1}$, el mayor número de turistas que visitaron el Perú en el año 2007 procedían de dicho país (11,1\%), mientras que tan solo un $45 \%$ de turistas provinieron de Francia. Así, se eligió al principal país de proveniencia de turistas (Esta-

\section{Gráfico 1}

Modelo conceptual

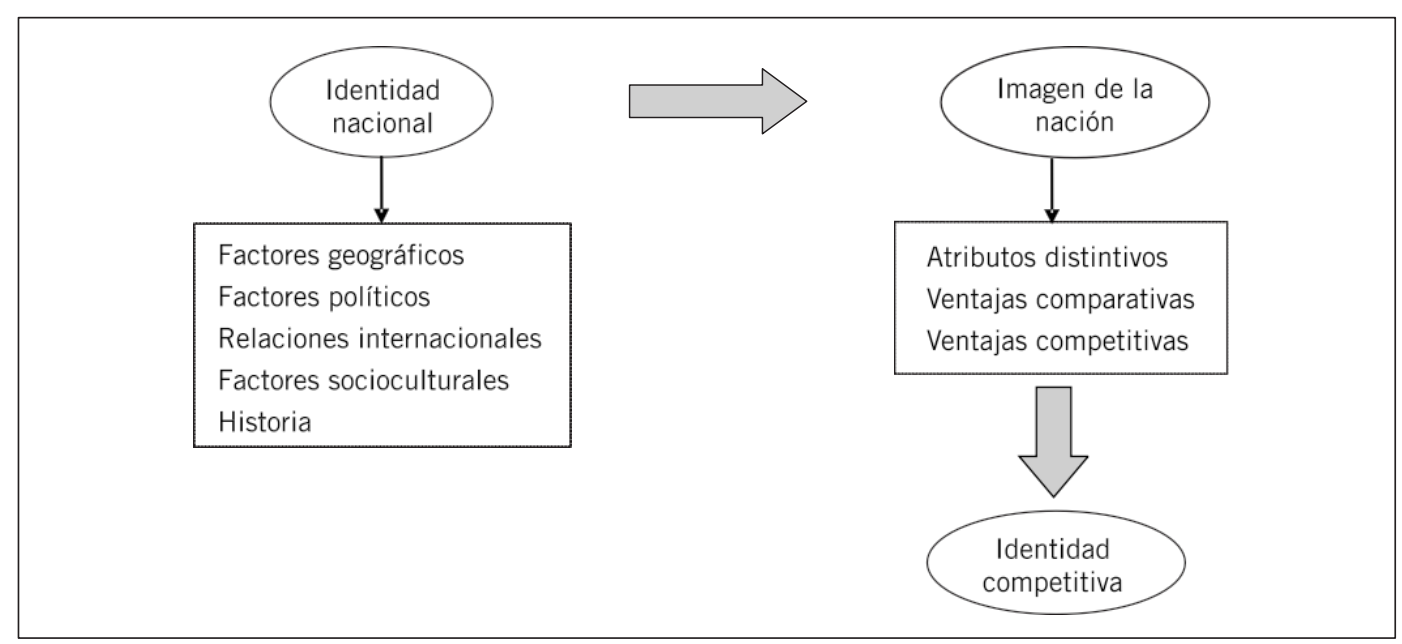

Fuente: Elaboración propia basada en el Modelo Identidad Marca País e Imagen de Dinnie (2008).

1. PromPerú es la organización del Gobierno peruano que promueve el comercio internacional de empresas peruanas. 
dos Unidos), a un país con poca proveniencia de turistas (Francia) y a los ciudadanos locales del país (Perú) para comparar la imagen que tenían del Perú los turistas extranjeros respecto de los pobladores locales.

Para la definición de la composición de la muestra, se consideraron las características sociodemográficas del perfil del turista extranjero que visita el Perú (PromPerú2), vale decir, personas con un nivel de educación superior, mayoritariamente hombres, cuya edad fluctúa fundamentalmente entre los 25 y 44 años.

Para la recolección de información, se aplicaron 356 encuestas, estructuradas con preguntas abiertas y cerradas al público de los tres países mencionados (véase el cuadro 1 ).

\section{Cuadro 1}

Composición de los encuestados por país de residencia

\begin{tabular}{|l|c|}
\hline País de residencia & Número de encuestados \\
\hline Perú & 122 \\
Estados Unidos & 119 \\
Francia & 115 \\
\hline TOTAL & $\mathbf{3 5 6}$ \\
\hline
\end{tabular}

Fuente: Elaboración propia sobre la base de los resultados de la investigación realizada.

\section{Discusión y análisis}

Los resultados preliminares de la parte empírica muestran que los consumidores evalúan el producto y sus atributos, extrínsecos e intrínsecos, en el proceso de decisión de compra, complementando su evaluación con el criterio del país de origen, el cual se utiliza a manera de información complementaria mas no exclusiva.

El análisis de los resultados de la imagen de la nación Perú entre los consumidores extranjeros, mostró que la imagen Perú no está asociada en absoluto con la oferta exportable peruana. La razón es que la marca Perú nunca se ha usado como paraguas para fomentar las exportaciones ni para incentivar la inversión, salvo el caso de la campaña «Perú Now», lanzada en el año 2008². Por lo tanto, su asociación es con elementos turísticos utilizados tradicionalmente en la estrategia de promoción de la marca Perú, como el caso de Machu Picchu, los incas, llamas, el Cusco, los Andes, entre otros elementos.

Por tal motivo, se requeriría incorporar determinados componentes de identidad nacional observados por el consumidor internacional, con el fin de promover la imagen de la nación del Perú entre los consumidores extranjeros. En definitiva, esos serán los elementos que determinen la identidad competitiva de un país, ya que el consumidor la identifica como elementos distintivos y diferenciadores frente al accionar y esfuerzos de otros países.

\section{Elementos que conforman la imagen de la nación Perú}

El cuadro 2 muestra los componentes de la imagen Perú, según la percepción de los encuestados. En dicho cuadro, se puede observar que los componentes de la imagen Perú están muy relacionados con el turismo, cuyos elementos son del tipo histórico-arqueológico.

Al analizar los resultados se evidencia el desconocimiento que existe de la amplia oferta y de la variedad de riquezas que tiene el Perú, dado que el grueso de los componentes de su imagen gira en torno al concepto histórico-arqueológico, asociado a la cultura Inca, mas no a sus otras riquezas, como la biodiversidad, sus culturas vivas, entre otros elementos turísticos. Cabe mencionar la falta de relación con la oferta exportable del Perú.

2. Perfil del Turista Extranjero 2007, disponible en:

http://www.peru.info/s_ftoPublicaciones.asp?HidAccion=Grupo\&Hidld=2\&ic=1\&SubTipo_ZP=1

3. «Perú Now» es una iniciativa privada, trabajada junto con el Gobierno peruano, para promover la captación de inversiones en el Perú con motivo de que el Perú es la sede de dos importantes cumbres internacionales, como son ALCUE y APEC. 
Cuadro 2

Componentes de la imagen de la nación Perú

\begin{tabular}{|l|c|r|}
\hline \multicolumn{3}{|c|}{ Imagen de la nación Perú } \\
\hline & $\mathbf{N}^{\circ}$ encuestados & \multicolumn{1}{c|}{$\%$} \\
\hline Machu Picchu & 80 & 22,5 \\
Llamas & 21 & 5,9 \\
Gastronomía & 20 & 5,6 \\
Incas & 20 & 5,6 \\
Cusco & 16 & 4,5 \\
Andes & 15 & 4,2 \\
Pisco & 13 & 3,7 \\
Lima & 11 & 3,1 \\
Ceviche & 10 & 2,8 \\
Lago Titicaca & 7 & 2,0 \\
Cultura & 7 & 2,0 \\
Otros & 136 & 38,2 \\
\hline TOTAL & $\mathbf{3 5 6}$ & $\mathbf{1 0 0 , 0}$ \\
\hline
\end{tabular}

Fuente: Elaboración propia sobre la base de los resultados de la investigación realizada.

La importancia de esta investigación radica en el hecho de que permite contrastar la estrategia para promocionar al país que ha venido aplicando el Perú durante los últimos años, con el posicionamiento generado en la mente de los consumidores.

Así, el concepto que tuvo mayor peso en la mención general fue el de Machu Picchu, que corresponde a su vez al ícono elegido por el Gobierno peruano para representar gráfica y visualmente al Perú, incluso desde antes de haber sido elegido como una de las nuevas siete maravillas del mundo.

Se observa, según los resultados empíricos obtenidos, que los componentes de imagen que están contenidos en la imagen del Perú no son adecuados para promover la oferta exportable peruana.

\section{Resultados}

En el cuadro 3 se muestra la distribución de respuestas respecto de la percepción de la imagen de la nación Perú en los ciudadanos de los tres países que integran la muestra: Francia, Estados
Unidos y Perú. De este modo, para Francia, un país no muy relacionado con el Perú, con escasos vínculos de turismo, cooperación económica y comercio exterior, la imagen de la nación predominantemente percibida por sus ciudadanos son las «llamas». Sin embargo, en el caso de los Estados Unidos y el Perú, sus ciudadanos relacionan su imagen, en primer lugar, con Machu Picchu. Además, es importante destacar que en el compendio total de respuestas de los tres países, la imagen Perú que se impone ante los demás conceptos es nuevamente la ciudadela incaica de Machu Picchu, con un 22,5\% del total, seguida de las «llamas» (que fue el ícono utilizado antes de Machu Pichu para la promoción del Perú) con un 5,9\% y, luego, la Gastronomía y los Incas, ambos con un 5,6\%.

\section{Cuadro 3}

Percepción de la imagen de la nación Perú

\begin{tabular}{|l|r|r|r|r|}
\hline \multirow{2}{*}{$\begin{array}{c}\text { Imagen Nación } \\
\text { Perú }\end{array}$} & \multicolumn{4}{|c|}{ Países } \\
\cline { 2 - 5 } & Francia & $\begin{array}{c}\text { Estados } \\
\text { Unidos }\end{array}$ & Perú & Total \\
\hline Machu Picchu & $12 \%$ & $26 \%$ & $26 \%$ & $22 \%$ \\
Llamas & $14 \%$ & $5 \%$ & $1 \%$ & $6 \%$ \\
Gastronomía & $0 \%$ & $3 \%$ & $12 \%$ & $6 \%$ \\
Incas & $8 \%$ & $4 \%$ & $5 \%$ & $6 \%$ \\
Cusco & $0 \%$ & $6 \%$ & $6 \%$ & $4 \%$ \\
Andes & $7 \%$ & $6 \%$ & $1 \%$ & $4 \%$ \\
Pisco & $1 \%$ & $4 \%$ & $5 \%$ & $4 \%$ \\
Lima & $10 \%$ & $1 \%$ & $0 \%$ & $3 \%$ \\
Ceviche & $0 \%$ & $1 \%$ & $6 \%$ & $3 \%$ \\
Lago Titicaca & $7 \%$ & $0 \%$ & $1 \%$ & $2 \%$ \\
Cultura & $1 \%$ & $2 \%$ & $2 \%$ & $2 \%$ \\
\hline Otros & $40 \%$ & $40 \%$ & $35 \%$ & $38 \%$ \\
\hline TOTAL & $100 \%$ & $100 \%$ & $100 \%$ & $100 \%$ \\
\hline
\end{tabular}

Fuente: Elaboración propia sobre la base de los resultados de la investigación realizada.

En el gráfico 2 se muestran los componentes de la imagen de la nación Perú, en él se puede observar la relación existente entre cinco de las seis primeras imágenes asociadas al Perú. Con excepción de la gastronomía, se presentan con mucha fuerza conceptos de origen andino/incaico asociados a la imagen Perú. Así, Machu Picchu, las llamas, Incas, Cusco y los Andes son concep- 
tos que se derivan del legado histórico, entre otras características propias de la cultura incaica.

La explicación de este último punto se debe al predominio en la orientación del mensaje e imagen del Perú, a lo largo de los años de campañas dirigidas al fomento del turismo, de su pasado incaico, representado tradicionalmente por la ciudadela de Machu Picchu en Cusco.

Sin embargo, se debe reconocer que entre estos conceptos vinculados a la herencia de culturas prehispánicas como la incaica, destaca uno que poco tiene que ver con este contexto y, por el contrario, se deriva de tradiciones ancestrales pero con elementos más contemporáneos: la gastronomía. Este componente, que para muchos encuestados de los tres países representa la imagen del Perú, ha recibido un impulso considerable a lo largo de los últimos tiempos, tanto por parte del Gobierno como del sector privado.
No obstante, al realizar el análisis comparativo de las percepciones de los consumidores de cada país estudiado, se observa que en Francia no se asocia el concepto de gastronomía a la imagen del Perú. Probablemente, porque ellos están muy orgullosos de su propia gastronomía.

Por otro lado, cabe señalar que, si bien no constituyen los principales elementos de la imagen del Perú, los conceptos de pisco y ceviche se encuentran incluidos como componentes de la imagen de la nación Perú.

Cabe destacar que la percepción de la imagen Perú, entre las tres diferentes muestras de Francia, Estados Unidos y Perú, determina una marcada percepción del Perú como destino turístico. Por ello, sería conveniente que la imagen de la nación asociada a una nación como el Perú, considerara los aspectos y las dimensiones asociados a la promoción de un país en un mundo global y competitivo.

\section{Gráfico 2}

Componentes de la imagen de la nación Perú

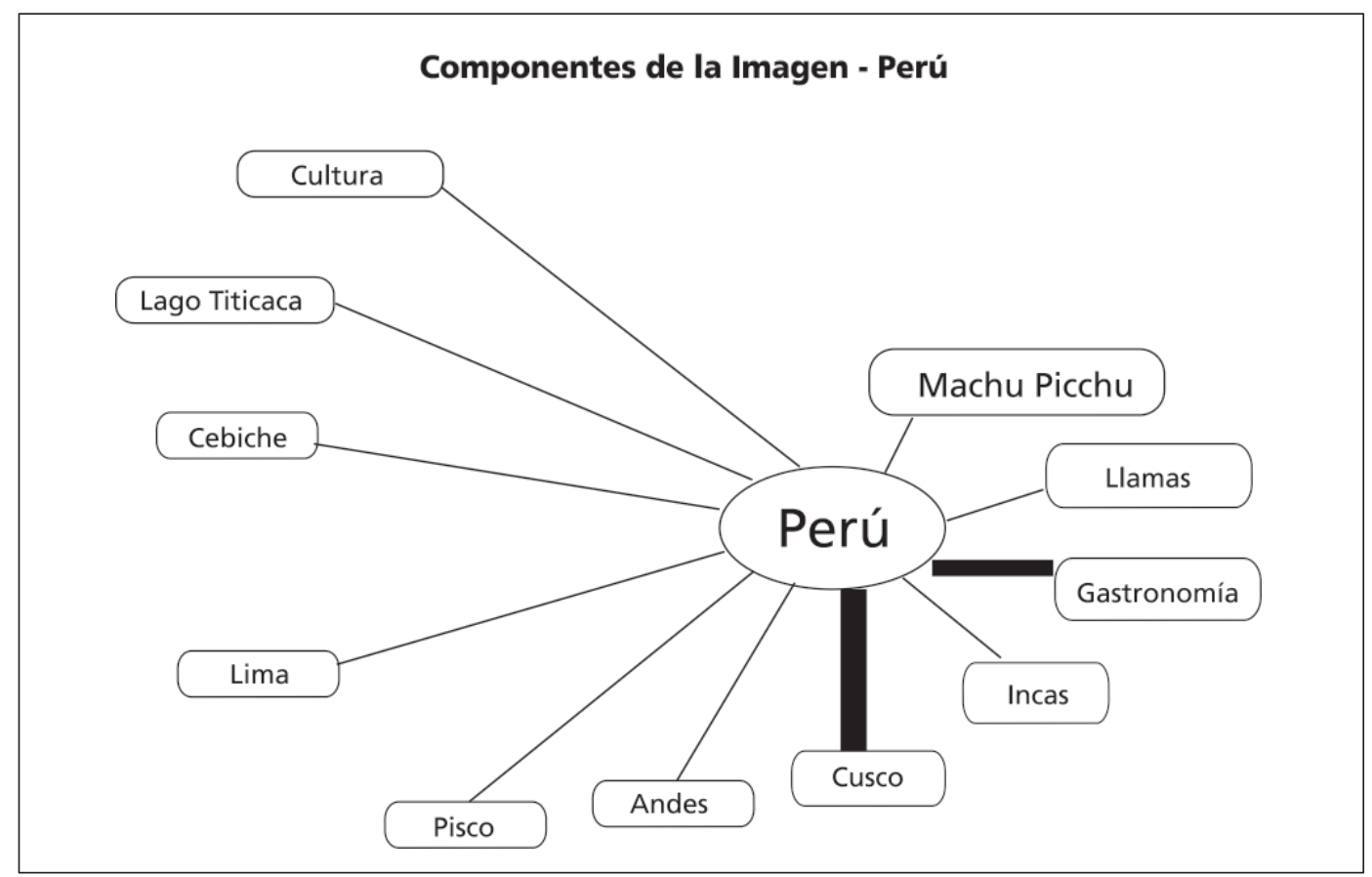

Fuente: Elaboración propia sobre la base de los resultados de la investigación realizada. 
La imagen de la nación del Perú que existe en la mente de los consumidores es la que se ha formado como resultado de estrategias deliberadas o no para alcanzar una ventaja competitiva del Perú, en la que se ha resaltado fundamentalmente el aspecto histórico, cultural y arqueológico del Perú mediante las campañas de promoción de su imagen realizadas por PromPerú: «Perú, país de los Incas», «Despierta tus seis sentidos», «Perú Ahora» $y$ «Perú: Vive la leyenda».

El problema radica en que en el Perú se ha ido cambiando con frecuencia el mensaje al consumidor, por lo que no se ha terminado de acuñar un único concepto en su mente. Tal como se puede apreciar en el gráfico 2, existen diversos conceptos que vienen a la mente de los consumidores cuando evocan la imagen del Perú, siendo la mayor parte de ellos alusivos al tema de los Incas (Machu Picchu, Incas, Ilamas, Cusco, Andes).

Esto se debe a que las dos primeras campañas, «Perú, país de los Incas», «Despierta tus seis sentidos», tuvieron una clara orientación al fomento del turismo en el país; mientras que la tercera, «Perú Ahora», se orientó a la captación de inversiones al país, con motivo de la celebración de las dos cumbres internacionales (ALCUE y APEC) que se llevarían a cabo en el Perú durante el año 2008. Finalmente, en el mes de octubre de 2008 se vuelve a retomar el tema del turismo, con la campaña: «Perú: Vive la leyenda».

Por ello, el Perú no cuenta con una imagen clara que trasmitir en sus campañas de comunicación, a pesar de contar con una riqueza invalorable de atributos diferenciadores respecto de otros mercados, como su oferta exportable, sus culturas vivas, su biodiversidad, sus hermosos paisajes naturales, entre otros.

Esto hace que sea necesario que el Perú replantee su estrategia de identidad competitiva y constru- ya una imagen clara y distintiva con otros países, que genere una ventaja competitiva, para poder posicionarse en la mente de los consumidores con un concepto específico que sirva para generar sinergias para sus tres objetivos principales: captar turistas, promover inversiones y fomentar las exportaciones.

\section{Conclusiones}

1. Los componentes de la imagen de la nación Perú están formados tanto por elementos turísticos, como por algunos elementos comerciales. Sin embargo, se observa el predominio de los atributos de índole turística por la estrategia de posicionamiento adoptada por el país.

2. Los componentes más importantes de la imagen de la nación Perú giran en torno al concepto de cultura milenaria y preinca. Se observa el predominio del ícono Machu Pichu, que ha sido utilizado como eje central de la comunicación del Perú para promover el turismo.

3. Otra categoría de componentes que se consideran relevantes para la imagen de la nación Perú es la referida a la cultura gastronómica oriunda del Perú, como elemento distintivo del país. Así, destacan los elementos de gastronomía, pisco y cebiche.

4. La cultura milenaria preinca e Inca, así como la cultura gastronómica, identificadas como elementos de la imagen nación Perú, deben ser tomadas en cuenta al momento de elaborar la estrategia de identidad competitiva del Perú, pues constituyen su ventaja diferencial.

5. No existen diferencias significativas entre las percepciones de los consumidores de los tres países analizados respecto de la imagen nación Perú, a pesar de tener distintos niveles de vinculación con nuestro país. 


\section{Referencias}

ANDERSON, B.

1991 Imagined Communities. Verso.

ANHOLT, Simon

2007 Competitive Identity: The New Brand Management for Nations, Cities and Regions. Reino Unido: Palgrave Macmillan.

BAUER, A., B. BLOCHING y K. HOWALDT

2006 Moment of Truth: Redefining the CEO's Brand Management Agenda. Reino Unido: Palgrave Macmillan.

\section{COMISIÓN DE PROMOCIÓN DEL PERÚ PARA LA} EXPORTACIÓN Y EL TURISMO (PromPerú)

s/f Perfil del Turista Extranjero 2007. Lima, Perú: PromPerú.

DINNIE, K.

2008 Nation Branding; Concepts, Issues, Practice. 1ra. edición. Reino Unido: ButterworthHeinemann.

HOWARD, Donald G.

1989 «Understanding how American Consumers Formulate their Attitudes about Foreign Products». En: Journal of International Consumer Marketing, Vol. 2, pp. 7- 24.

JAWORSKI, S.P. y FOSHER D.

2003 «National brand identity \& its effect on corporate brands: The nation brand effect (NBE)». En: Multinational Business Review, Vol. 11, pp. 99-113.

KELLER, K.L.

2003 Strategic Brand Management: Building, Measuring, and Managing Brand Equity. Segunda edición. Estados Unidos: Prentice Hall.

KOTLER, Philip y David GERTNER

2002 «Country as Brand, product, and beyond: A place marketing and brand management». En: Journal of Brand Management, Vol. 9, pp. 4$5,249-261$.
KOTLER, Philip; D.H. HAIDER e I. REIN

1993 Marketing Places: Attracting Investment, Industry and Tourism to Cities, States and Nations. En: Free Press, USA.

LECLERE, France; Bernd H. SCHMITT y Laurette DUBÉ

1994 « Foreign Branding and Its Effects on Product Perceptions and Attitudes». En: Journal of Marketing Research, Vol. 31, mayo, pp. 263270.

MADHAVARAM, S.; V. BADRINARAYANAN y R.E. MC DONALD

2005 «Integrated Marketing Communication and Brand Identity as Critical Components of Brand Equity Strategy: A conceptual framework and research propositions». En: Journal of Advertising, Vol. 34, pp. 68- 80.

OLINS, W.

2002 «Branding the Nation-the historical context». En: Journal of Brand Management, Vol. 9, pp. 4- 5, 241-248 .

MAHOMAD, Osman; Zafar U. AHMED, Earl D. HONEYCUTT JR y Taizoon Hyder TYEBKHAN

2000 «Does «made in...» matter to consumers? A Malaysian study of country of origin effect». En: Multinational Business Review; Vol. 8 , ABI/INFORM Global.

O'SHAUGHNESSY, J. y N. JACKSON

2000 «Treating the Nation as a Brand: Some Neglected Issues». En: Journal of Macromarketing, Vol. 20, pp. 56-64.

PORTER, Michael

1998 The Competitive Advantage of Nations. Reino Unido: Palgrave.

SMITH, A.D.

1991 National Identity. Londres: Penguin Books. 
Los componentes de la imagen de la nación como base para formulación de la identidad competitiva: el caso del Perú

SHULMAN, S.

2002 «Challenging the civic/ ethnic and west/ east dichotomies in the study of nationalism». En: Comparative Political Studies, Vol. 35, pp. 554-585.

\section{TEMPORAL, P.}

2002 Advanced Brand Management: From Vision to Valuation. Singapur, Asia: John Wiley y Sons.
TUCK, J.

2003 «The Men in White». En: International Review for the Sociology of Sport, Vol. 38, pp.177199.

VANOSSI, P.

2006 «Country as Brand: Nation Branding», disponible en http://www.affisch.org/weblog/archives/00000187.html. 